\title{
La atención especializada del Sistema Nacional de Salud durante la Gran Recesión. Gasto, utilización y variabilidad en el periodo 2004-2015
}

\author{
Salvador Peiró* \\ Manuel Ridao* \\ Fundación para el Fomento de la Investigación Sanitaria y Biomédica \\ de la Comunidad Valenciana (FISABIO)
}

\section{Resumen}

Reducir las variaciones en la práctica médica (VPM) es un objetivo implícito de los sistemas sanitarios financiados públicamente. En este trabajo se describe la evolución del gasto hospitalario y las hospitalizaciones en el Sistema Nacional de Salud (SNS), totales y por determinadas condiciones características de atención efectiva, discrecional y sensible a la oferta, durante los periodos 2004-2009 y 2010-2015, analizando sus tendencias y variabilidad entre áreas de salud. Durante 2010-2015, el SNS incrementó notablemente su eficiencia a expensas de incrementar su actividad y la complejidad de su casuística mientras se mantenía estabilizado el gasto global, pero no se redujo la variabilidad en utilización y gasto entre áreas de salud.

Palabras clave: utilización hospitalaria, gasto hospitalario, variaciones en la práctica médica.

Clasificación JEL: H51, I11, I12.

\begin{abstract}
Reducing medical practice variation is an implicit objective of publicly funded healthcare systems. This paper describes hospital expenditure and hospitalization evolution in the Spanish National Health System (SNS), total and by certain characteristic conditions of effective, discretionary and supply-sensitive care, during the period 2004-2009 and 2010-2015, analyzing their trends and variability between health areas. During 2010-2015, the SNS notably increased its efficiency at the expense of increasing its activity and case-mix complexity while global spending was stabilized, although unwarranted variations between health areas did not decrease.
\end{abstract}

Keywords: hospital utilization, hospital expenditure, medical practice variations.

JEL classification: H51, I11, I12.

* Los autores pertenecen al Grupo Atlas de Variaciones en la Práctica Médica en el Sistema Nacional de Salud y a la Red de Investigación en Servicios de Salud en Enfermedades Crónicas (REDISSEC) y, en el caso de Manuel Ridao, al Instituto Aragonés de Ciencias de la Salud (IACS). 


\section{Introducción}

Cuántas veces, cuándo, por qué motivos o por cuánto tiempo las personas son hospitalizadas o tratadas, qué pruebas diagnósticas y qué tratamientos reciben, a qué coste y qué resultados obtienen en términos de supervivencia y calidad de vida son cuestiones críticas para cualquier sistema sanitario. Es llamativo que la respuesta a estas preguntas parece depender más del lugar en que las personas residen que de cuán enfermas están (Peiró y Maynard, 2015). A nivel poblacional, la geografía -antes que la morbilidad- es el principal determinante de la utilización de servicios sanitarios (sean intervenciones quirúrgicas, hospitalizaciones, pruebas diagnósticas, visitas a urgencias o a consulta, tratamientos farmacológicos u otros numerarios de utilización) y del gasto en salud. Y, al contrario de lo que sería esperable, al menos en los países desarrollados, los territorios con mayor utilización o mayor gasto sanitario no parecen alcanzar mejores resultados en salud (Fisher et al., 2003a; 2003b).

Las variaciones «injustificadas» (unwarranted variations) en la práctica médica (VPM) han sido definidas como aquellas variaciones que no pueden ser explicadas por diferencias en la prevalencia de enfermedad, la evidencia científica o las preferencias de los pacientes (Wennberg, 2010). Desde los trabajos iniciales de Wennberg y colegas (Wennberg y Gittelshon, 1973, 1982; Wennberg et al., 1982) numerosos estudios en prácticamente todos los países desarrollados han demostrado la presencia de notables variaciones injustificadas en la práctica médica (Corallo et al., 2014), incluidos los países que cuentan con sistemas de atención tipo National Health Service como Reino Unido (NHS England, 2018) o España (Atlas VPM, 2018; Bernal-Delgado et al., 2014).

Los hallazgos cardinales de estas investigaciones muestran que las VPM son extensas, sistemáticas (no debidas al azar), persistentes en el tiempo, apenas atenuadas tras ajustar por diferencias socio-demográficas, y sin correlación entre el gasto y la calidad de la atención (Sirovich et al., 2006). Más allá de la preocupación por el despilfarro o las posibles desigualdades en el acceso a la atención sanitaria, el mensaje más desconcertante de las VPM es que los profesionales sanitarios tratan de forma muy diferente a pacientes muy similares, quebrando la creencia tradicional de que aplican de forma uniforme un tratamiento inequívocamente apropiado («científico», «basado en la evidencia») a los mismos problemas de salud (Peiró y Maynard, 2015).

Durante la Gran Recesión, el Sistema Nacional de Salud español (SNS) estuvo sujeto a importantes presiones presupuestarias. Según la Estadística de Gasto Sanitario Público publicada por el Ministerio de Sanidad (MSBCS, 2018), el gasto sanitario total (en euros corrientes) creció desde 38,6 millones de euros en 2002 hasta 70,7 millones de euros en 2009, iniciando un descenso en 2010 (año que puede considerarse el inicio de la reducción presupuestaria en sanidad) hasta los 61,9 millones de euros de 2014 (reducción de algo más del 12 por 100 respecto a 2009), volviendo a la senda ascendente en 2015 para situarse en 2016 en 66,7 millones de euros, una cifra similar a la de 2008. Aunque el descenso fue menos acusado en la atención especializada (término que incluye la atención hospitalaria de cualquier 
tipo y la atención especializada extrahospitalaria) que en la atención primaria o en la salud pública, un reciente trabajo señalaba que el gasto por habitante en el SNS creció desde 441 euros en 2003 hasta 643 euros en 2009, para caer hasta 576 euros en 2013 e iniciar un nuevo ascenso hasta igualar en 2015 las cifras de 2009 (cifras en euros constantes de 2003; Ridao-López et al., 2018). Ese mismo trabajo también sugería que la variabilidad en gasto en atención especializada por habitante entre las áreas de salud del SNS creció durante la reciente crisis económica.

Fisher y Wennberg (2003) desarrollaron una taxonomía de las tecnologías e intervenciones sanitarias en función de las causas atribuibles a su variabilidad y sus implicaciones. La «atención efectiva» incluiría aquellas intervenciones o tratamientos para los que existe una sólida evidencia científica de su eficacia en una condición clínica concreta. Este tipo de intervenciones mostrarían una variabilidad reducida sugiriendo una posible subutilización en las áreas de menor consumo (los pacientes candidatos no recibirían un servicio sanitario que sería efectivo en su situación). Ejemplos arquetípicos de atención efectiva serían la hospitalización por fractura de cadera o por apendicitis aguda. La «atención discrecional» $\mathrm{o}$ «sensible a las preferencias de los pacientes» sería aquella para la que se dispone de evidencia inconcluyente o los posibles cursos de acción se asocian a diferentes resultados con un amplio espacio para que pacientes distintos elijan razonablemente alternativas diferentes en función de sus preferencias. Las VPM en estos casos son difíciles de interpretar, pero tenderían a indicar un escaso ajuste de los profesionales sanitarios a las preferencias de los pacientes. La literatura suele ejemplificarlos con procedimientos de cirugía electiva como la colecistectomía o la artroplastia de rodilla. La «atención sensible a la oferta» correspondería a tecnologías o servicios caracterizados por la escasez de evidencias sobre su valor en circunstancias clínicas concretas, con importantes discrepancias sobre su indicación y que presentan tasas de utilización asociadas positivamente a la disponibilidad de recursos. Las VPM en este último caso sugerirían sobreutilización de servicios sanitarios (Peiró et al., 2005) y los arquetipos serían la amigdalectomía o las hospitalizaciones por condiciones potencialmente evitables.

Aunque no es posible establecer una tasa «idónea» de utilización de servicios sanitarios (Frohlich, Roos, 1982), reducir las VPM -especialmente las que se deben a sub y sobreutilización- es un objetivo implícito de los sistemas sanitarios financiados públicamente y uno de sus indicadores de calidad más relevantes (Wennberg, 2011). El objetivo de este trabajo es describir la evolución del gasto hospitalario y las hospitalizaciones en el SNS, totales y por determinadas condiciones características de atención efectiva, discrecional y sensible a la oferta, durante los periodos 2004-2009 y 2010-2015 (seis años antes y seis años después del inicio del impacto de la Gran Recesión sobre el SNS que, orientándose por la primera reducción en los presupuestos sanitarios, se ha situado en 2010). 


\section{Población y métodos}

\subsection{Diseño}

Estudio poblacional (ecológico), descriptivo del gasto en atención especializada y las tasas de ingresos hospitalarios, totales y por seis condiciones seleccionadas, en las áreas de salud del SNS.

\subsection{Población y ámbito}

Todas las áreas de salud del SNS $(n=203)$ excepto las ciudades autónomas de Ceuta y Melilla. La mayor parte de estas áreas de salud cuentan con una población de entre 150.000 y 250.000 residentes servidos por un hospital de la red del SNS y las consultas externas y centros de especialidades dependientes del mismo. La atención primaria se organiza en Zonas Básicas de Salud, en su mayor parte entre 5.000 y 25.000 residentes, que se suman territorialmente para configurar el área de salud. Debido a sus características organizativas (planificación geográfica, escasas barreras de accesibilidad y ausencia de incentivos económicos a los proveedores), los pacientes reciben la mayor parte de la atención no terciaria en su área de salud de residencia. Los criterios de selección para las poblaciones incluidas en los diversos análisis (todos los ingresos, ingresos por apendicectomía, fractura de cadera en personas de 50 y más años, colecistectomías en personas de 20 y más años, artroplastia de rodilla en personas de 45 y más años, amigdalectomía con o sin adenoidectomía en personas de menos de 20 años y hospitalizaciones potencialmente evitables en personas de 40 y más años) se recogen en el Anexo.

\subsection{Fuentes de datos}

Los datos demográficos proceden de los censos municipales mantenidos por el Instituto Nacional de Estadística que fueron agregados por áreas de salud. Los datos de hospitalizaciones proceden del Proyecto Atlas de Variaciones en la Práctica Médica en el Sistema Nacional de Salud que, a su vez, proceden del Conjunto Mínimo de Datos Básicos al Alta Hospitalaria de los servicios regionales de salud de las diferentes Comunidades Autónomas, y fueron cedidos ya agrupados por áreas de salud y grupos etarios. Los datos de gasto, también agregados por áreas de salud, proceden de la Estadística de Centros Sanitarios de Atención Especializada (ECSAP) para 2010-2015, y de la Estadística de Establecimientos Sanitarios en Régimen de Internado (EESRI) hasta 2010 (La EESRI fue reformada ese año para incorporar la actividad y gasto de los centros de especialidades extrahospitalarios y transformada en la ECSAP). 


\subsection{Variables y definiciones}

Se utilizaron las siguientes variables y definiciones:

- Gasto en atención especializada (o gasto hospitalario): sumatorio del gasto anual consolidado de los hospitales del SNS y centros de especialidades asociados. Los términos «atención especializada» y «hospitalario» se utilizan como sinónimos en todo el texto, y siempre incluyen la atención especializada bajo ingreso y externa. Cuando se divide entre el número de habitantes ofrece el gasto hospitalario por habitante.

- Hospitalización: episodios de ingreso hospitalarios para la atención de pacientes y episodios de Cirugía Mayor Ambulatoria (CMA). Una misma persona puede tener varios episodios al año. Cuando se divide entre el número de habitantes, se presenta como tasa de hospitalización por 100 habitantes, también llamada tasa de frecuentación hospitalaria. Cuando se refiere a procedimientos específicos se emplearon tasas por 10.000 habitantes.

- Razón de variación (RV). Razón de gasto hospitalario por habitante (o de ingresos hospitalarios por habitante) entre las áreas de salud con mayor y menor gasto por habitante (o mayor y menor tasa de hospitalización). Para limitar el efecto de algunos valores outlier se utilizan los valores de las áreas de salud en los percentiles 95 (P95) y 5 (P5). Se utiliza como indicador grosero de variabilidad. Una RV5-95 de, por ejemplo, 4 se interpreta como que el área en el P95 tiene un gasto por habitante cuatro veces mayor -o un 300 por 100 mayor- que el área de salud en el P5 (Librero et al., 2009).

- Componente Sistemático de la Variación (CSV): mide la variación de la desviación entre la tasa observada y esperada, expresada como porcentaje de la tasa esperada. Es una medida derivada a partir de un modelo que reconoce dos fuentes de variación: variación sistemática (diferencia entre áreas) y variación aleatoria (diferencia dentro de cada área). A mayor CSV mayor variación sistemática (no esperable por azar).

\subsection{Aspectos éticos}

El estudio -observacional, utilizando las áreas de salud como unidad de análisis y realizado con datos retrospectivos previamente anonimizados de forma irreversible y transferidos a los investigadores ya agrupados por áreas de salud y grupos etarios- no utilizó datos personales identificados o identificables y, conforme a las regulaciones españoles e internacionales, no requiere aprobación por un Comité de Ética de Investigación. 


\subsection{Análisis}

En primer lugar, se describió la evolución anual de la población (en millones de habitantes), gasto hospitalario total (en millones de euros, sin deflactar) y volumen de ingresos hospitalarios (en millones de ingresos) y se utilizaron regresiones lineales segmentadas para comparar las tendencias de crecimiento entre los sexenios 2004-2009 (que se considera previo al impacto de la Gran Recesión en el sector salud) y 2010-2015 (periodo de recesión). Los valores de $p$ en la sección de resultados corresponden a la significación estadística del cambio de tendencia respecto a la del periodo previo. El punto de corte se fijó en 2010 considerando que hasta este año -y pese a lo avanzado de la crisis económica- el gasto sanitario público no había sufrido recortes sustantivos y continuaba creciendo a un ritmo ajeno al de otros sectores ya muy afectados por la Gran Recesión. También se analizó el porcentaje de actividad (ingresos hospitalarios) realizados para actividad obstétrica.

En segundo lugar, se analizó la evolución anual del gasto hospitalario por habitante y de las tasas de hospitalización y la variabilidad de ambas variables entre áreas de salud. Para calcular estos parámetros se utilizó el gasto corriente y las tasas de hospitalización crudas (sin estandarizar por edad y sexo) del correspondiente año. Para el análisis de variaciones se utilizó la RV5-95. Al igual que en el caso anterior se utilizaron modelos de regresión segmentada para identificar cambios en la tendencia de crecimiento entre los dos sexenios. En estos análisis los pacientes fueron contabilizados en su área de residencia (no necesariamente la del hospital donde recibieron atención). Secundariamente se utilizó el test de Pearson para analizar las correlaciones bivariables entre las tasas de frecuentación en los diferentes años y el gasto por habitante en los diferentes años.

Finalmente se analizó la variabilidad en las tasas de hospitalización estandarizadas por edad y sexo para las condiciones previamente descritas. La estandarización se realizó por el método indirecto respecto a la distribución por grupos quinquenales de edad y sexo de la población española de 2003 y las tasas resultantes pueden interpretarse como las tasas de hospitalización que habrían tenido las diferentes áreas de salud en el respectivo año si hubieran tenido una población con una distribución por edad y sexo similar a la del conjunto de la población española de 2003.

\section{Resultados}

Durante el periodo 2004-2009, la población española creció de 43,1 a 46,6 millones de habitantes (incremento del 8 por 100), y continuó creciendo hasta 2012 para descender a partir de esa fecha y situarse en los 46,5 millones de habitantes en 2015, con un sustancial incremento de los mayores de 65 años (de 7,3 a 8,6 millones de personas, incremento del 18 por 100). La regresión segmentada muestra que el crecimiento poblacional se produjo sobre todo en el periodo 2004-2009 (incremento medio de 0,69 millones de habitantes/año; $p<0,001)$, mientras que la tendencia cambió en el periodo 2010-2015 ( $-0,79$ millones/año respecto a la pendiente del 
sexenio previo; $p<0,001)$, lo que se tradujo en un estancamiento del crecimiento durante este segundo periodo (Cuadro 1 y Gráfico 1 ).

El gasto en atención especializada del SNS creció desde 20.573 millones de euros en 2004 hasta 35.089 millones de euros en 2011 (incremento del 71 por 100), para descender por debajo de los 34.000 millones de euros de 2012 a 2014 y ascender de nuevo hasta los 36.112 millones de euros en 2015. El modelo de regresión segmentada muestra que durante 2004-2009 el gasto hospitalario total creció a una media de 2.722 millones de euros/año $(p<0,001)$, para invertir esta tendencia en 20102015 (-2.578 millones de euros/año respecto a la tendencia del periodo 2004-2009; $p<0,001)$, con el resultado de una tendencia de crecimiento prácticamente estancada en este segundo periodo (Gráfico 1).

El número total de altas de hospitalización creció desde 4,13 millones en 2004 a 5,22 millones en 2015 (incremento del 26 por 100). Este incremento sería algo mayor (en torno al 32 por 100) si se excluyen las altas obstétricas, un aspecto relevante ya que la natalidad descendió ostensiblemente durante la Gran Recesión y la actividad obstétrica supone una parte muy importante de la actividad hospitalaria (el 11,6 por 100 en 2004 frente al 7,6 por 100 en 2015). El crecimiento medio anual del periodo 2004-2009 fue de 0,12 ingresos por 100 habitantes $(p<0,001)$, tendencia que se modifica durante 2010-2015, con una reducción de 0,05 ingresos/100 habitantes respecto al periodo previo $(p=0,044)$ que atenuó la pendiente de crecimiento de la utilización hospitalaria durante este periodo (Gráfico 1).

\section{CUADRO 1}

POBLACIÓN, GASTO EN ATENCIÓN ESPECIALIZADA

Y HOSPITALIZACIONES 2004-2015

\begin{tabular}{|c|c|c|c|c|c|c|c|c|c|c|c|c|}
\hline & 2004 & 2005 & 2006 & 2007 & 2008 & 2009 & 2010 & 2011 & 2012 & 2013 & 2014 & 2015 \\
\hline \multicolumn{13}{|c|}{ Población total (en millones de habitantes) } \\
\hline Población & 43,1 & 44,0 & 44,8 & 45,3 & 46,1 & 46,6 & 46,9 & 47,0 & 47,1 & 46,9 & 46,6 & 46,5 \\
\hline \multicolumn{13}{|c|}{ Gasto total en atención especializada (en millones de euros) } \\
\hline Gasto & 20.573 & 22.889 & 25.029 & 27.928 & 31.512 & 33.873 & 34.017 & 35.089 & & & & 36.111 \\
\hline \multicolumn{13}{|c|}{ Número de ingresos hospitalarios totales (en millones de hospitalizaciones) } \\
\hline Hospitalizaciones & 4,13 & 4,30 & 4,42 & 4,58 & 4,67 & 4,76 & 4,81 & 4,85 & 4,82 & 4,91 & 5,06 & 5,22 \\
\hline \multicolumn{13}{|c|}{ Gasto por habitante: mediana de las áreas de salud y valores en las áreas en el P5 y P95 (en euros) } \\
\hline Gasto/habitante & 484,3 & 525,9 & 568,4 & & 697,8 & 744,9 & 756,5 & & 732,4 & & & 802,2 \\
\hline P5 & & 379 & & & & 553 & & & & & & 547,8 \\
\hline & & 703 & 735 & & & 989,3 & $1.055,2$ & & & 954,2 & & $1.118,8$ \\
\hline RV5-95 & 1,82 & 1,86 & 1,74 & 1,68 & 1,72 & 1,79 & 2,00 & 2,01 & 2,00 & 1,88 & 1,97 & 2,04 \\
\hline \multicolumn{13}{|c|}{ Ingresos hospitalarios por 100 habitantes: mediana de las áreas de salud y valores en las áreas en el P5 y P95 } \\
\hline Tasa hospit. & 10,08 & 10,25 & 10,36 & 10,45 & 10,55 & 10,35 & 10,42 & 10,16 & 9,91 & 10,15 & 10,20 & 10,61 \\
\hline P5 & 7,27 & 7,25 & 7,19 & 7,24 & 7,25 & 7,43 & 7,27 & 7,42 & 7,19 & 7,57 & 7,68 & 8,01 \\
\hline P95 & 12,48 & 12,55 & 13,13 & 13,24 & 13,22 & 13,31 & 13,73 & 13,99 & 14,13 & 14,50 & 14,84 & 14,74 \\
\hline RV5-95 & 1,72 & 1,73 & 1,83 & 1,83 & 1,82 & 1,79 & 1,89 & 1,89 & 1,97 & 1,92 & 1,93 & 1,84 \\
\hline
\end{tabular}

FUENTE: Elaboración propia.

NOTA: P5: tasa del área de salud en el percentil 5 de la respectiva distribución; P95: tasa del área de salud en el percentil 95 de la respectiva distribución; RV5-95: razón entre la tasa del área en el P5 y en el P95. 


\section{GRÁFICO 1 \\ POBLACIÓN, GASTO HOSPITALARIO Y HOSPITALIZACIONES \\ EN EL SISTEMA NACIONAL DE SALUD (2004-2015). NÚMERO Y MODELOS \\ DE REGRESIÓN SEGMENTADA 2004-2009 Y 2010-2015}
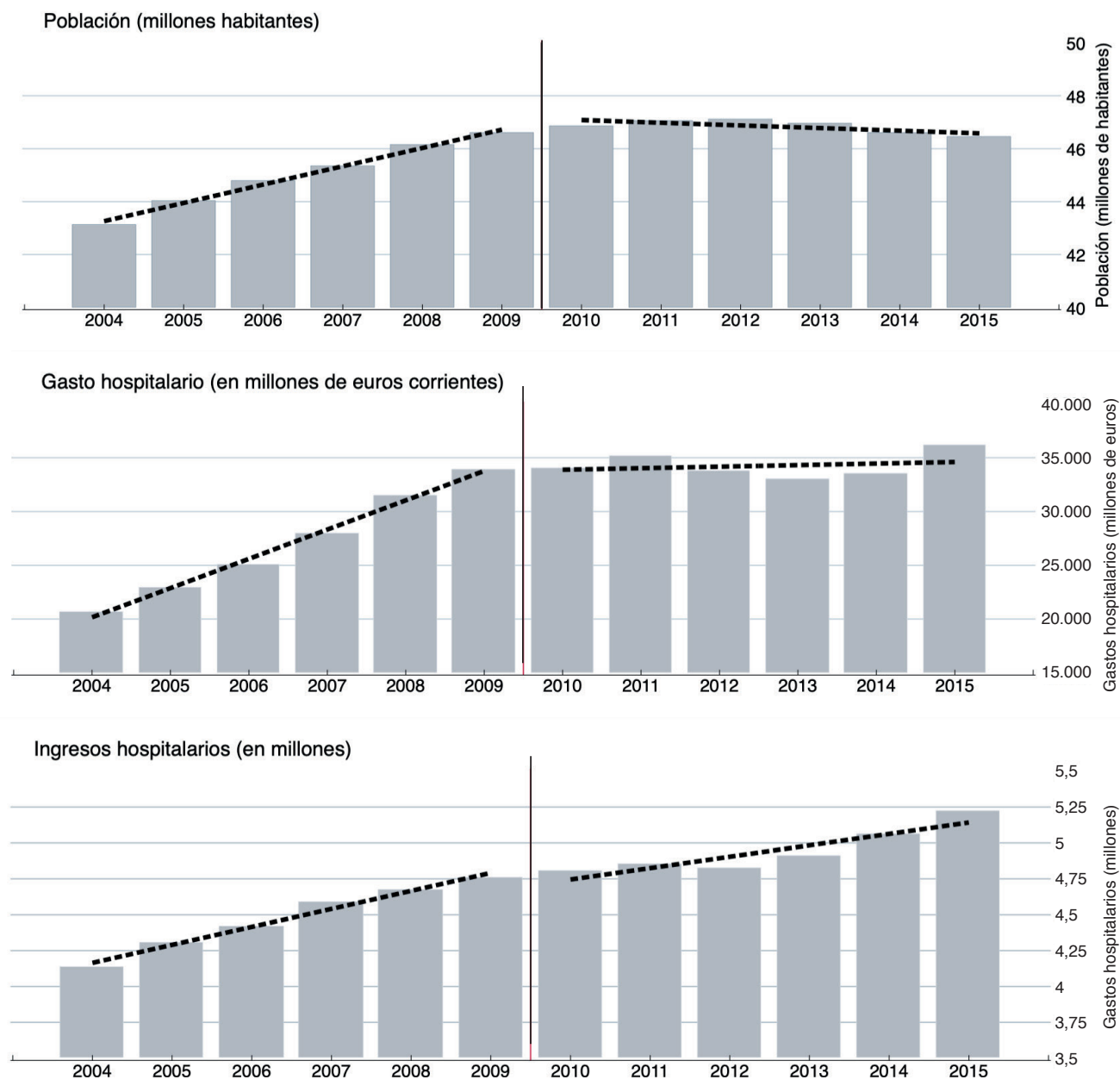

FUENTE: Elaboración propia. 


\section{GRÁFICO 2}

GASTO HOSPITALARIO POR HABITANTE Y TASAS DE HOSPITALIZACIÓN POR 100 HABITANTES EN EL SISTEMA NACIONAL (2004-2015)

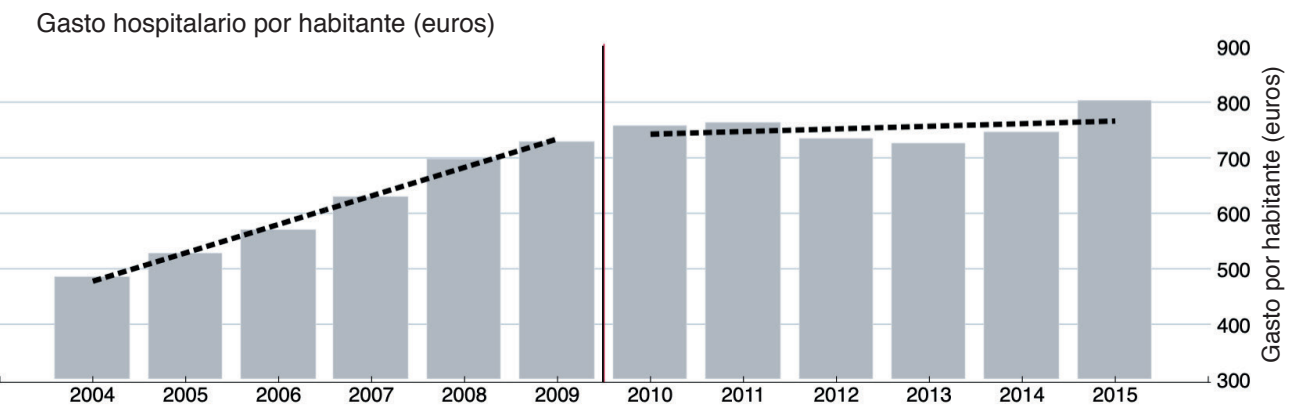

Gasto hospitalario por habitante (euros/año) en las áreas en el P5 y P95

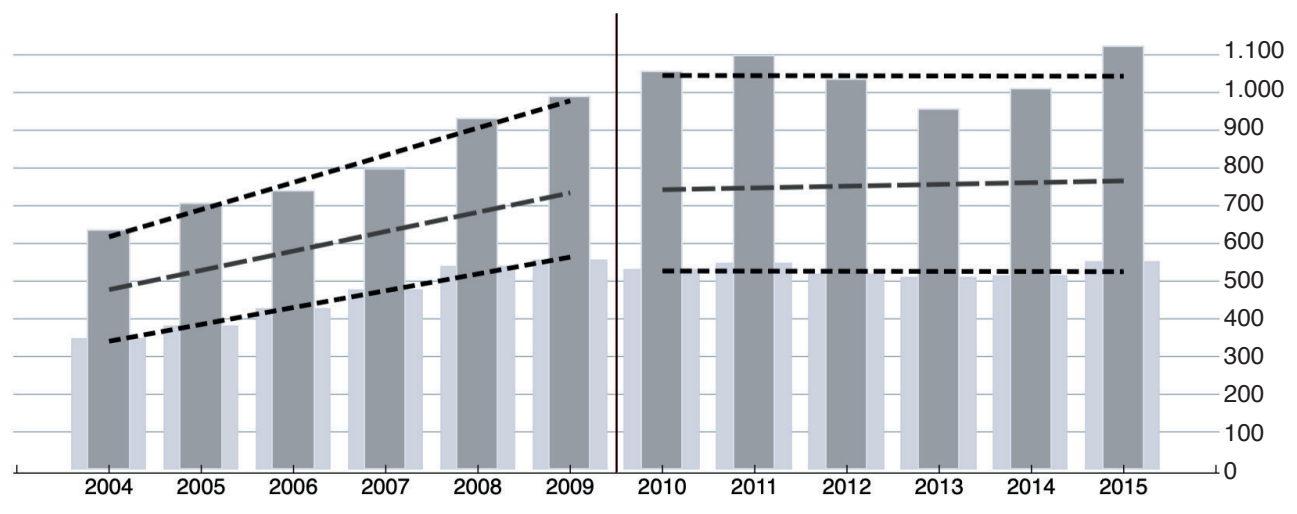

Hospitalizaciones por 100 habitantes

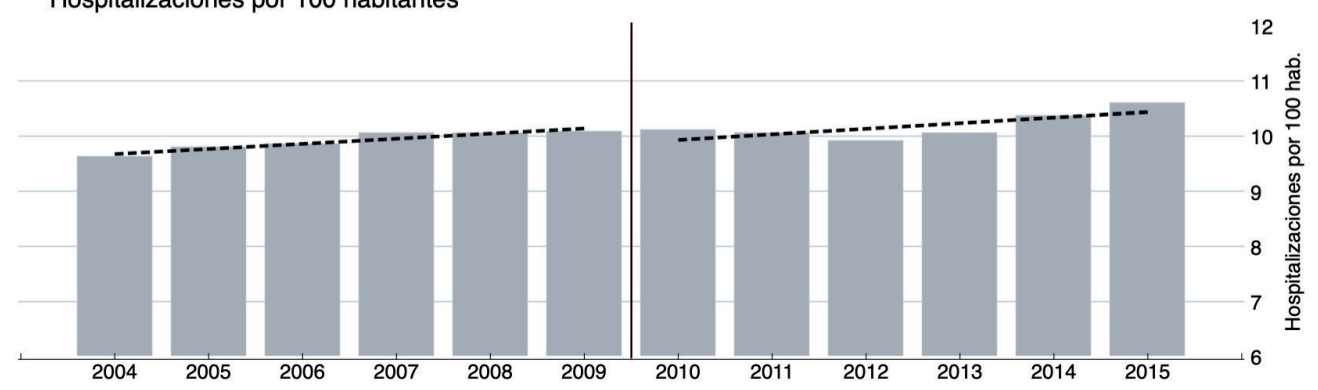

FUENTE: Elaboración propia. 


\section{GRÁFICO 2 (Continuación)}

\section{GASTO HOSPITALARIO POR HABITANTE Y TASAS DE HOSPITALIZACIÓN POR 100 HABITANTES EN EL SISTEMA NACIONAL (2004-2015)}

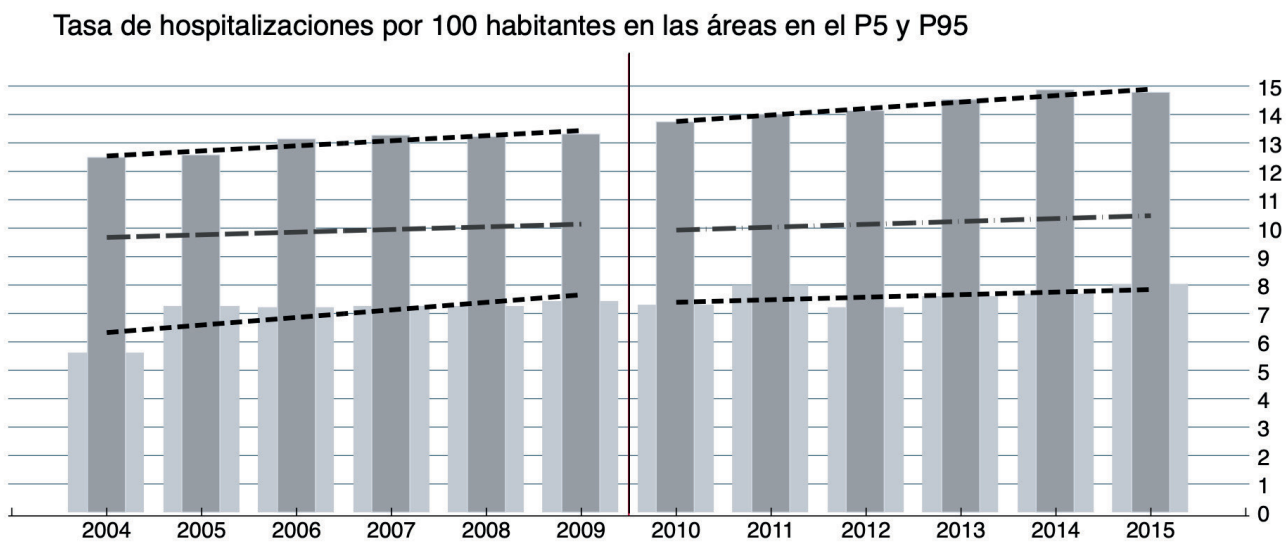

FUENTE: Elaboración propia.

El gasto hospitalario por habitante creció desde 484 euros en 2004 hasta 763 euros en 2011, para mantenerse en torno a los 730 euros en los tres años siguientes y ascender a 802 euros en 2015 (incremento del 66 por 100 respecto a 2004), con una tendencia ascendente entre 2004-2009 (crecimiento anual medio de 51 euros/habitante; $p<0,001$ ) que cambió en el siguiente sexenio (descenso anual medio de 47 euros/habitante respecto a la tendencia previa; $p<0,001)$ con lo que prácticamente permaneció estabilizada en este segundo periodo (Cuadro 1 y Gráfico 2). La variabilidad en gasto hospitalario por habitante entre áreas de salud, se mantuvo estable durante 2004-2009 (en torno a un 80 por 100 más gasto por habitante en el área en el P95 respecto al área de salud en el P5), aun con un descenso medio anual de 0,02 puntos (p: 0,260). En 2010-2015, y sobre una constante 0,25 puntos mayor que en el periodo previo (p: 0,008), el gasto en el área en el P95 dobló el del área en el P5, pero la tendencia no mostró cambios significativos (incremento anual medio de 0,02 puntos respecto al periodo previo; $p$ : 0,412).

Las tasas de hospitalización crecieron desde 9,6 ingresos por 100 habitantes en 2004 hasta 10,0 en 2007, manteniéndose estables entre 9,9 y 10,1 hasta 2014, momento en que vuelven a crecer hasta alcanzar los 10,6 ingresos por 100 habitantes en 2015 (incremento del 8,3 por 100 respecto a 2004). El incremento medio anual fue de 0,01 ingresos por 100 habitantes en el sexenio 2004-2009 ( $p: 0,023)$ y sin cambios significativos respecto a esta tendencia en el periodo 2010-2015 (Cuadro 1 y Gráfico 2). La variabilidad en las tasas de ingresos entre las diferentes áreas de salud se mantuvo relativamente estable durante todo el periodo estudiado, con tasas de ingreso en torno a un 80 por 100 mayores en el área en el P95 respecto al área en el 
P5. Los modelos de regresión segmentada no detectaron cambios significativos entre periodos en la razón de utilización entre las áreas en el P5 y P95.

La correlación en el gasto por habitante de las áreas de salud a lo largo de los años (datos no presentados) es muy elevada. En general, superaba el 0,80 para los años inmediatamente siguientes al tomado como referencia y se mantenía por encima del 0,40-0,50 en los años más distantes de la serie. Del mismo modo, la correlación entre las tasas de hospitalización de las áreas de salud a lo largo del tiempo fue también muy elevada, con coeficientes de correlación en torno a 0,80-0,90 en los años inmediatos y en torno al 0,30-0,40 en los más distantes. La correlación entre gasto y tasas de hospitalización, aun importante, es menos elevada, con coeficientes de correlación por debajo de 0,50 en los años inmediatos al de referencia y por debajo de 0,20 en los más lejanos.

En el Cuadro 2 y el Gráfico 3 se muestra el número de hospitalizaciones, las tasas estandarizadas por edad y sexo (media del SNS y tasa de las áreas en el P5 y el P95) y los estadísticos de variabilidad (razón entre la tasa del área en el P95 y la del P5, y componente sistemático de la variación, CSV) de las seis condiciones analizadas. Las dos condiciones seleccionadas como «atención efectiva» (apendicectomías y hospitalizaciones por fractura de cadera) mantuvieron tasas relativamente estables durante todo el periodo y una variabilidad reducida (CSV por debajo de 0,10 todos los años). El número de fracturas de cadera aumentó de poco más de 39.000 a casi 50.000 por el envejecimiento de la población, pero la tasa estandarizada por edad y sexo se redujo discretamente y también la variabilidad entre áreas de salud.

De las condiciones seleccionadas como «sensibles a las preferencias de los pacientes» por tratarse de cirugía electiva, la colecistectomía se comportó de forma similar a las condiciones de «atención efectiva», con un notable incremento de intervenciones (de poco más de 50.000 a casi 66.000 entre 2004 y 2015) pero tasas relativamente estables durante todo el periodo (aun con un incremento desde 15,1 a 16,5 intervenciones por 10.000) y reducida variabilidad. La artroplastia de rodilla, al contrario, mostró un notable incremento en la tasa de intervenciones (de 21,1 a 26,3 intervenciones por 10.000) y una mayor variabilidad (entre 3 y 5 veces más intervenciones en el área en el P95 respecto al área en el P5) que se redujo durante el periodo analizado.

De las condiciones seleccionadas como «sensibles a la oferta», la tasa de amigdalectomías se mantuvo estable durante el periodo, siempre con una gran variabilidad entre áreas (entre seis y diez veces más intervenciones en el área en el P95 respecto al área en el P5), mientras que las hospitalizaciones potencialmente evitables continuaron el descenso iniciado previamente al comienzo de la Gran Recesión, pasando de tasas de 80,3 por 10.000 en 2004 a 60,5 por 10.000 en 2015. La variabilidad entre áreas (en torno a tres veces más ingresos en el área en el P95 respecto al área en el P5) también se redujo. 
CUADRO 2

HOSPITALIZACIONES POR DIVERSAS CONDICIONES EN EL SISTEMA NACIONAL DE SALUD (2004-2015). TASAS ESTANDARIZADAS POR EDAD Y SEXO Y VARIABILIDAD ENTRE ÁREAS DE SALUD

\begin{tabular}{|c|c|c|c|c|c|c|c|c|c|c|c|c|}
\hline & 2004 & 2005 & 2006 & 2007 & 2008 & 2009 & 2010 & 2011 & 2012 & 2013 & 2014 & 2015 \\
\hline \multicolumn{13}{|c|}{ Apendicectomías por $\mathbf{1 0 . 0 0 0}$ hab. Todas las edades } \\
\hline $\mathrm{n}$ & 43.493 & 43.547 & 43.939 & 44.278 & 45.468 & 46.807 & 46.268 & 45.631 & 43.980 & 44.326 & 43.978 & 43.079 \\
\hline Tasa Est. & 10,65 & 10,43 & 10,06 & 10,11 & 10,24 & 10,48 & 10,33 & 10,21 & 9,86 & 9,99 & 9,98 & $\mathbf{9 , 8 3}$ \\
\hline Tasa P05 & 7,02 & 6,99 & 6,81 & 6,97 & 7,12 & 7,19 & 7,25 & 7,02 & 6,93 & 6,97 & 7,07 & 7,05 \\
\hline Tasa P95 & 16,11 & 16,30 & 16,47 & 16,26 & 16,28 & 15,82 & 16,27 & 16,33 & 15,44 & 15,77 & 15,40 & 14,64 \\
\hline EQ5-95 & 2,29 & 2,33 & 2,42 & 2,33 & 2,29 & 2,20 & 2,24 & 2,33 & 2,23 & 2,26 & 2,18 & 2,08 \\
\hline CSV & 0,07 & 0,08 & 0,09 & 0,09 & 0,09 & 0,07 & 0,07 & 0,09 & 0,08 & 0,06 & 0,07 & 0,06 \\
\hline \multicolumn{13}{|c|}{ Fractura de cadera por 10.000 hab. $>39$ años } \\
\hline $\mathrm{n}$ & 39.122 & 40.595 & 41.485 & 43.274 & 44.217 & 44.349 & 46.440 & 45.859 & 46.639 & 47.673 & 48.312 & 49.948 \\
\hline Tasa Est. & 29,93 & 29,79 & 29,59 & 29,43 & 29,31 & 27,90 & 29,09 & 27,65 & 27,33 & 26,84 & 27,20 & 27,54 \\
\hline Tasa P05 & 13,99 & 14,14 & 13,97 & 16,45 & 14,26 & 14,29 & 16,64 & 14,56 & 16,73 & 16,99 & 17,41 & 16,07 \\
\hline Tasa P95 & 40,41 & 40,09 & 38,53 & 38,63 & 39,11 & 37,00 & 37,94 & 37,12 & 35,28 & 35,61 & 35,40 & 35,40 \\
\hline EQ5-95 & 2,89 & 2,84 & 2,76 & 2,35 & 2,74 & 2,59 & 2,28 & 2,55 & 2,11 & 2,10 & 2,03 & 2,20 \\
\hline CSV & 0,08 & 0,08 & 0,08 & 0,07 & 0,06 & 0,07 & 0,06 & 0,06 & 0,07 & 0,05 & 0,05 & 0,04 \\
\hline \multicolumn{13}{|c|}{ Colecistectomías por 10.000 hab. > 19 años } \\
\hline $\mathrm{n}$ & 50.084 & 51.192 & 53.315 & 54.725 & 56.903 & 59.616 & 62.365 & 62.777 & 62.406 & 64.603 & 66.444 & 65.889 \\
\hline Tasa Est. & 15,11 & 15,20 & 14,78 & 14,90 & 15,21 & 15,70 & 16,28 & 16,12 & 15,89 & 16,36 & 16,78 & 16,53 \\
\hline Tasa P05 & 8,71 & 8,47 & 9,66 & 8,92 & 8,71 & 9,95 & 10,02 & 10,48 & 9,91 & 10,92 & 11,83 & 11,36 \\
\hline Tasa P95 & 21,62 & 23,23 & 23,19 & 22,03 & 22,46 & 24,18 & 23,69 & 23,39 & 24,27 & 23,57 & 23,89 & 24,42 \\
\hline EQ5-95 & 2,48 & 2,74 & 2,40 & 2,47 & 2,58 & 2,43 & 2,36 & 2,23 & 2,45 & 2,16 & 2,02 & 2,15 \\
\hline CSV & 0,07 & 0,08 & 0,09 & 0,07 & 0,07 & 0,08 & 0,06 & 0,06 & 0,07 & 0,06 & 0,05 & 0,07 \\
\hline \multicolumn{13}{|c|}{ Artroplastia de rodilla por 10.000 hab. $>44$ años } \\
\hline $\mathrm{n}$ & 35.100 & 38.254 & 40.278 & 41.730 & 42.933 & 43.842 & 43.427 & 42.476 & 42.468 & 45.909 & 49.816 & 49.510 \\
\hline Tasa Est. & 21,12 & 21,95 & 23,23 & 23,88 & 24,37 & 24,57 & 24,21 & 23,26 & 23,14 & 24,92 & 26,89 & 26,35 \\
\hline Tasa P05 & 7,21 & 8,13 & 9,99 & 10,20 & 11,54 & 10,51 & 10,30 & 11,30 & 10,25 & 12,35 & 12,94 & 12,31 \\
\hline Tasa P95 & 39,61 & 44,86 & 45,91 & 45,88 & 45,58 & 44,03 & 43,02 & 41,06 & 41,39 & 42,40 & 48,96 & 47,01 \\
\hline EQ5-95 & 5,49 & 5,52 & 4,60 & 4,50 & 3,95 & 4,19 & 4,18 & 3,63 & 4,04 & 3,43 & 3,78 & 3,82 \\
\hline CSV & 0,27 & 0,25 & 0,26 & 0,25 & 0,23 & 0,21 & 0,20 & 0,18 & 0,19 & 0,16 & 0,19 & 0,22 \\
\hline \multicolumn{13}{|c|}{ Amigdalectomías con/sin adenoidectomía por 10.000 hab. $<20$ años } \\
\hline $\mathrm{n}$ & 11.446 & 12.167 & 12.732 & 13.672 & 14.287 & 14.683 & 14.047 & 14.148 & 13.914 & 14.135 & 14.447 & 13.404 \\
\hline Tasa Est. & 13,87 & 13,29 & 12,67 & 13,01 & 14,10 & 13,70 & 13,15 & 13,53 & 13,33 & 13,17 & 13,76 & 12,54 \\
\hline Tasa P05 & 3,47 & 3,21 & 4,07 & 4,22 & 4,79 & 4,61 & 3,68 & 5,11 & 4,88 & 4,51 & 3,54 & 2,74 \\
\hline Tasa P95 & 28,18 & 27,94 & 32,72 & 31,08 & 29,39 & 32,64 & 31,18 & 30,23 & 29,35 & 30,31 & 30,33 & 28,78 \\
\hline EQ5-95 & 8,12 & 8,70 & 8,04 & 7,36 & 6,14 & 7,08 & 8,47 & 5,92 & 6,01 & 6,72 & 8,57 & 10,50 \\
\hline CSV & 0,46 & 0,35 & 0,31 & 0,40 & 0,34 & 0,29 & 0,34 & 0,43 & 0,38 & 0,40 & 0,30 & 0,39 \\
\hline \multicolumn{13}{|c|}{ Hospitalizaciones potencialmente evitables por 10.000 hab. > 39 años } \\
\hline $\mathrm{n}$ & 162.693 & 171.257 & 159.219 & 172.321 & 161.436 & 158.241 & 154.748 & 151.922 & 152.340 & 146.456 & 146.728 & 152.097 \\
\hline Tasa Est. & 80,27 & 82,52 & 77,35 & 79,21 & 74,11 & 67,78 & 65,62 & 63,68 & 63,14 & $\mathbf{5 9 , 8 0}$ & 60,21 & 60,52 \\
\hline Tasa P05 & 45,28 & 44,47 & 38,65 & 41,54 & 38,01 & 35,16 & 32,11 & 32,74 & 34,41 & 31,41 & 29,77 & 32,37 \\
\hline Tasa P95 & 135,23 & 136,51 & 127,05 & 133,95 & 113,71 & 116,03 & 112,10 & 101,08 & 99,69 & 88,40 & 88,10 & 89,40 \\
\hline EQ5-95 & 2,99 & 3,07 & 3,29 & 3,22 & 2,99 & 3,30 & 3,49 & 3,09 & 2,90 & 2,81 & 2,96 & 2,76 \\
\hline CSV & 0,14 & 0,14 & 0,14 & 0,14 & 0,11 & 0,11 & 0,13 & 0,12 & 0,11 & 0,12 & 0,10 & 0,10 \\
\hline
\end{tabular}

FUENTE: Elaboración propia.

NOTA: P05: tasa del área de salud en el percentil 5 de la respectiva distribución; P95: tasa del área de salud en el percentil 95 de la respectiva distribución; EQ:5-95: razón entre la tasa del área en el P5 y en el P95; CSV: componente sistemático de la variación. 


\section{GRÁFICO 3}

HOSPITALIZACIONES POR DIVERSAS CONDICIONES EN EL SISTEMA NACIONAL DE SALUD (2004-2015). TASAS ESTANDARIZADAS

\section{POR EDAD Y SEXO}

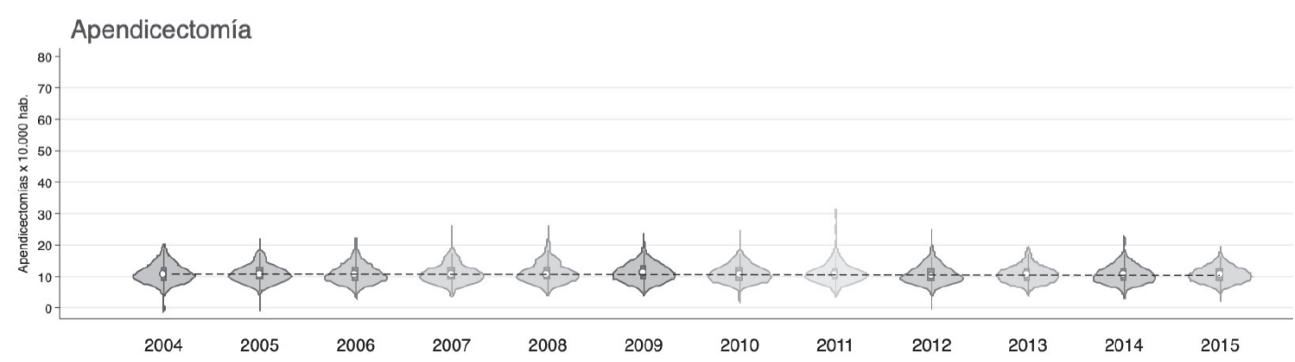

Fractura de cadera
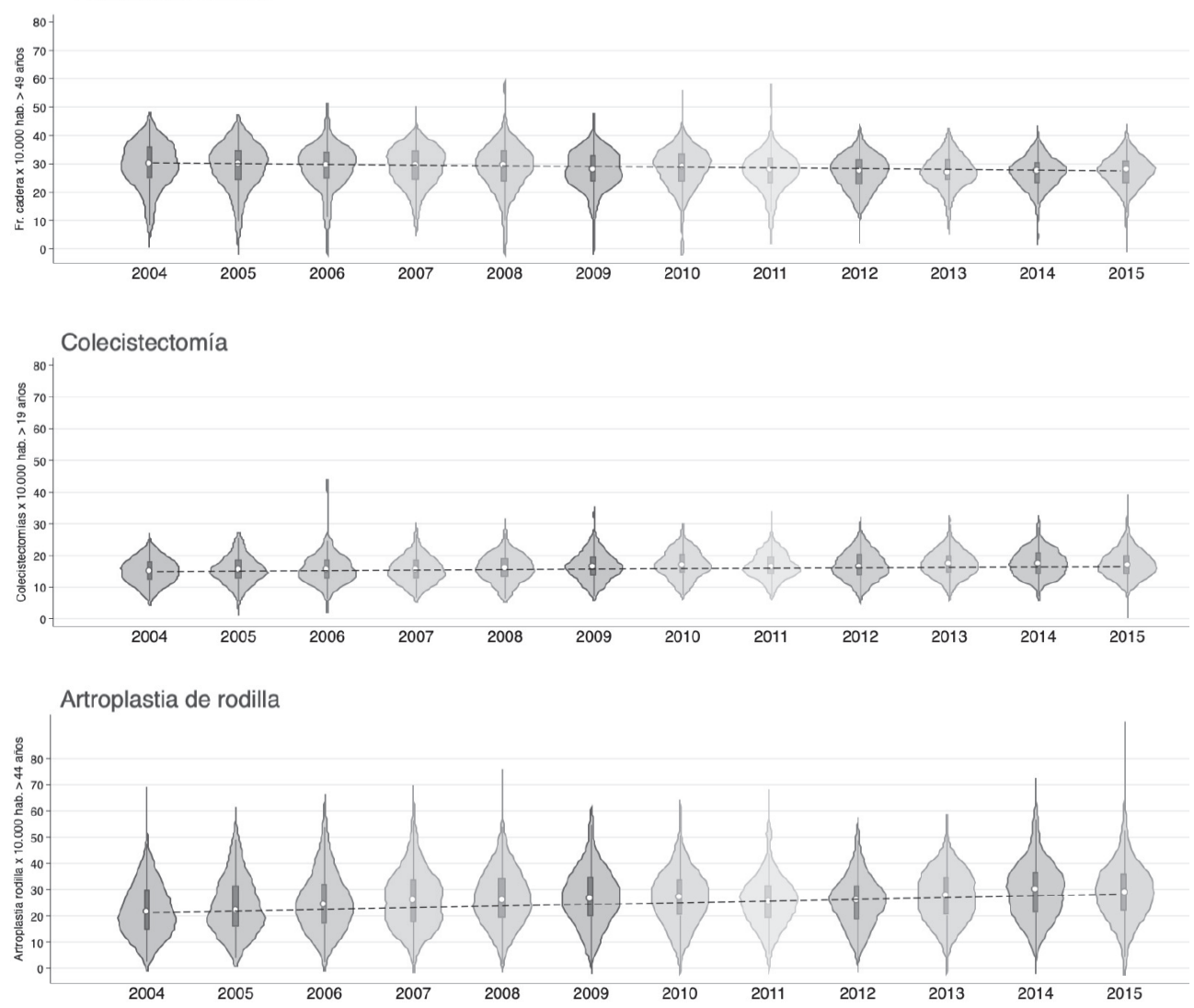

FUENTE: Elaboración propia. 


\section{GRÁFICO 3 (Continuación)}

\section{HOSPITALIZACIONES POR DIVERSAS CONDICIONES EN EL SISTEMA NACIONAL DE SALUD (2004-2015). TASAS ESTANDARIZADAS POR EDAD Y SEXO}
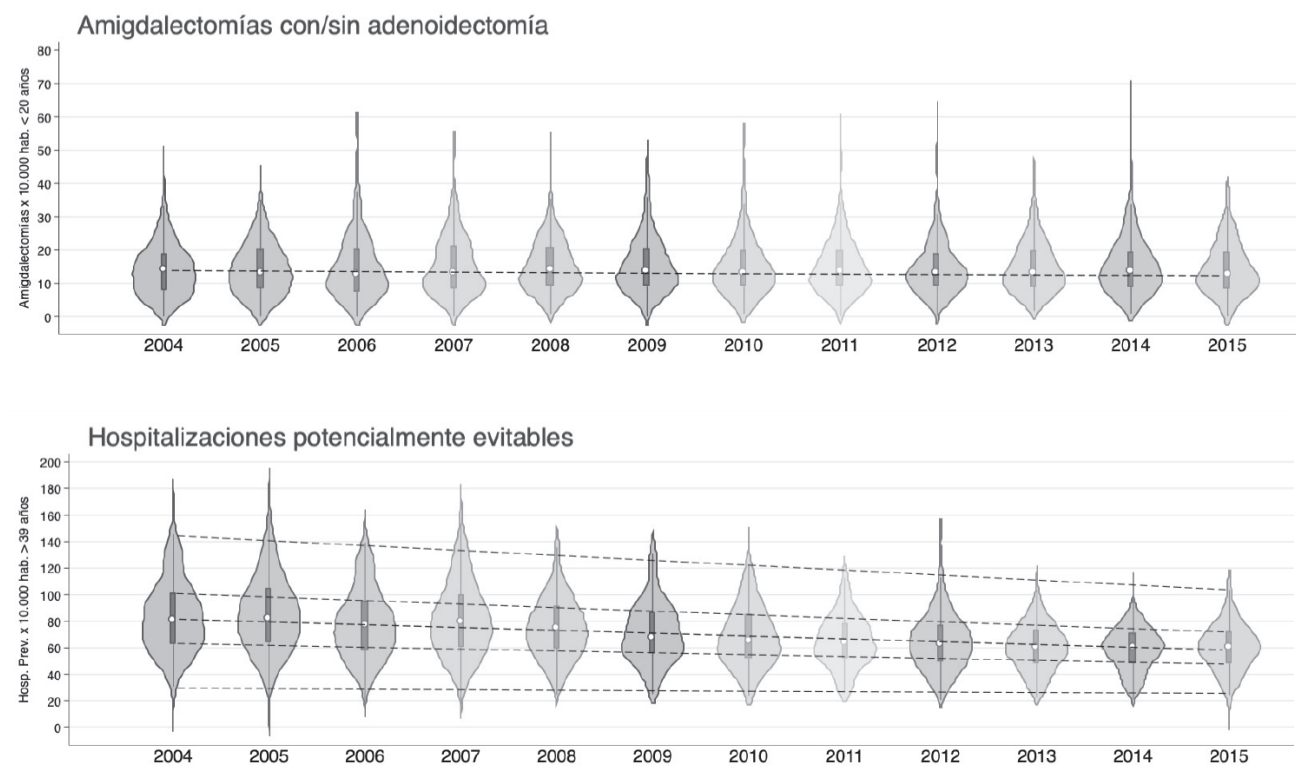

FUENTE: Elaboración propia.

\section{Discusión}

Durante el periodo 2010-2015, tanto el volumen de población como el gasto hospitalario total, que venían de un intenso crecimiento en el periodo 2004-2009, pasaron a mantenerse relativamente estables en las cifras iniciales del periodo $\mathrm{y}$, en consecuencia, el gasto hospitalario por habitante se mantuvo también estable en torno a los 750 euros por habitante y año. Esta relativa estabilidad en el gasto hospitalario global se produce pese al notable incremento del gasto en farmacia hospitalaria (Ministerio de Sanidad, Consumo y Bienestar Social, 2018) cuyo crecimiento, en la práctica, fue financiado con la reducción del gasto de personal (derivado de la reducción de salarios y del volumen de personal) para mantener el presupuesto nivelado en las cifras iniciales del sexenio hasta 2015. El volumen de hospitalizaciones, sin embargo, continuó creciendo a un ritmo similar al del periodo 2004-2009 (más de 400.000 ingresos adicionales en 2015 respecto a 2010), a lo que cabría añadir un aumento en la complejidad de la casuística por la reducción de la proporción de ingresos de menor complejidad (descenso en partos y reducción de hospitalizaciones potencialmente evitables). Este incremento en el volumen de ingresos se trasladó a un incremento muy discreto de las tasas estandarizadas de hospitalización (con un 
leve descenso al inicio del sexenio y un ascenso más marcado en los dos últimos años), por lo que cabe inferir que derivó fundamentalmente del envejecimiento poblacional.

La variabilidad entre áreas de salud, tanto en gasto por habitante como en hospitalizaciones por habitante se mantuvo relativamente estable durante ambos sexenios en cifras en torno al doble de gasto (y doble de ingresos) en las áreas de salud con mayor gasto (o utilización) respecto a las que menos. Frente a la posibilidad de que durante el periodo 2010-2015, las áreas con mayor gasto hubieran atenuado su crecimiento en mayor proporción que las áreas con menos recursos, el gasto hospitalario creció -o decreció- en la misma proporción en ambos tipos de áreas, manteniéndose las desigualdades en niveles muy similares durante todo el periodo estudiado. En general, estos resultados serían coincidentes con otros trabajos recientes que han analizado la utilización y el gasto en el SNS (Ridao-López et al., 2018; Gorgemans et al., 2018).

Respecto a las condiciones específicas, tanto la apendicectomía como las hospitalizaciones por fractura de cadera (atención efectiva en terminología de VPM, cuya reducción hubiera supuesto dejar sin atención condiciones vitales) mostraron tasas estables, absorbiendo con los mismos recursos el importante incremento en el número de fracturas de cadera (desde 39.000 a 50.000) debido al envejecimiento de la población. El volumen de colecistectomías (50.000 en 2004, 59.600 en 2009 y casi 66.000 en 2015) y el de artroplastias de rodilla (35.000 en 2004, casi 44.000 en 2009 y 49.500 en 2015) aumentó notablemente en el periodo estudiado, aunque el envejecimiento de la población, como en el caso de la fractura de cadera, atemperó el crecimiento de las tasas estandarizadas. En cuanto a las condiciones sensibles a la oferta, el volumen de amigdalectomías, que había crecido en el periodo previo a la crisis económica (desde casi 11.400 a 14.700), se redujo hasta 13.400 intervenciones, mientras el volumen de hospitalizaciones potencialmente evitables, que venía descendiendo desde su pico máximo en 2007 (172.000 ingresos), y pese al envejecimiento poblacional que debería afectar de este tipo de hospitalizaciones fundamentalmente crónicas, se redujo hasta 152.000. La variabilidad tendió a reducirse discretamente en todos los casos (salvo la colecistectomía que se mantuvo estable).

En conjunto, la Gran Recesión, con su correlato de restricciones presupuestarias en el SNS desde 2010, no se acompañó de una reducción de la utilización hospitalaria. Bien al contrario, el SNS -y pese a la ausencia de crecimiento de recursosabsorbió el incremento de volumen derivado del envejecimiento de la población para mantener estables las tasas poblacionales de atención efectiva y sensible a las preferencias de los pacientes, absorbió el incremento del gasto farmacéutico hospitalario y de la complejidad de la casuística atendida y creció en cirugía electiva. Todo ello sin reducir la variabilidad entre áreas de salud en gasto por habitante o en hospitalizaciones por habitante. La reducción en hospitalizaciones potencialmente evitables -en su mayor parte descompensaciones de enfermedades crónicas- puede interpretarse como un incremento adicional de la eficiencia, bien porque se redujeron poblacionalmente, bien -y más probable- por el desarrollo (ya iniciado en los 
años previos a la crisis) de nuevos modelos de manejo de las descompensaciones y exacerbaciones de los pacientes crónicos (sin ingreso, resolución en observación de urgencias, hospitalización a domicilio) que permitieron reducir el recurso a la hospitalización convencional.

Un aspecto también destacable es la serendipidad del sistema de atención especializada del SNS, con presupuestos anuales por área de salud que son explicados hasta en un 90 por 100 por el gasto del año previo, y hasta en un 50-60 por 100 por el gasto de hace diez años. No es un fenómeno extraño en una organización cuyo principal componente de gasto, los recursos humanos, tienen carácter para-funcionarial y los variables acaban dependiendo de la incorporación, en mayor o menor medida, de nuevos y costosos medicamentos (oncológicos, biológicos, para enfermedades huérfanas). En este estudio los costes de los pacientes con estas condiciones han sido referidos al área de residencia, pero su atención se concentra -más que razonablemente- en los grandes hospitales terciarios y, en la práctica, el gasto por centros habrá crecido más en estos últimos que en los hospitales medianos y pequeños (con una proporción menor de gasto de farmacia y mayor de gastos de personal).

El actual estudio tiene varias limitaciones. En primer lugar, los sexenios elegidos incluyen un cambio en la metodología de las fuentes de información sobre gasto (en 2010 la EESRI se transformó en ECSAP para unificar el gasto de los centros de especialidades con el de los hospitales). Aunque la partida de los centros de especialidades es menor en el conjunto del gasto hospitalario, es previsible que de mantenerse la serie inalterada el descenso del gasto hospitalario en el segundo sexenio hubiera sido algo mayor. En segundo lugar, para los análisis globales, se construyeron series crudas (no estandarizadas), por lo que el gasto o las hospitalizaciones por habitante no son directamente comparables ni entre ejercicios (que incluyen poblaciones cada vez más envejecidas), ni entre áreas de salud (que pueden incluir desde áreas con poblaciones jóvenes y baja morbilidad a áreas muy envejecidas).

En tercer lugar, aunque la población ha sido contabilizada siempre en su área de residencia (no en la del hospital donde se hospitalizó al paciente) puede existir un cierto grado de mala clasificación, especialmente en las áreas servidas por hospitales terciarios. En cuarto lugar, la población censal es diferente a la población cubierta por el SNS, especialmente por el colectivo de funcionarios del Estado atendidos por las mutualidades públicas que mayoritariamente eligen recibir la atención sanitaria a través de aseguradoras privadas. Aunque no es previsible que esto influya en las tendencias temporales, los análisis transversales de variabilidad entre áreas pueden quedar afectados si algunas de ellas incluyen porcentajes muy diferentes de este tipo de funcionarios (sería una población, concentrada en la capital del Estado, contabilizada en el denominador de las tasas pero que no consumiría recursos del SNS). Finalmente, la atención hospitalaria presta muchos otros servicios además de la hospitalización (consultas externas, pruebas diagnósticas, atención urgente, sesiones de hospital de día, sesiones de quimioterapia, diálisis, rehabilitación, intervenciones menores, etc., además de las asociadas a la investigación y la docencia) que no se han considerado en este estudio y que conforman una parte cada vez más importante 
de la actividad hospitalaria. Tampoco se han considerado otros aspectos relevantes como la calidad de la atención hospitalaria.

\section{Conclusiones}

En conjunto, y con las limitaciones señaladas previamente, durante el sexenio 2010-2015, el SNS incrementó notablemente su eficiencia a expensas de incrementar su actividad y la complejidad de su casuística mientras se mantenía estabilizado el gasto global. Este incremento de actividad le permitió mantener las tasas de hospitalización absorbiendo el crecimiento derivado del envejecimiento poblacional. La variabilidad entre áreas de salud, salvo para las hospitalizaciones potencialmente evitables, no se redujo en el periodo 2010-2015 de modo que, aun sobre un gasto total estancado, las diferencias entre áreas de salud se mantuvieron. El territorio de residencia continúa siendo un determinante clave en la utilización de servicios sanitarios.

\section{Referencias bibliográficas}

[1] BERNAL-DELGADO, E.; GARCÍA-ARMESTO, S.; PEIRÓ, S.; ATLAS VPM GROUP (2014). «Atlas of Variations in Medical Practice in Spain: the Spanish National Health Service under scrutiny». Health Policy, 114 (1), 15-30.

[2] CORALLO, A. N.; CROXFORD, R.; GOODMAN, D. C.; BRYAN, E. L.; SRIVASTAVA, D. y STUKEL, T. A. (2014). «A systematic review of medical practice variation in OECD countries». Health Policy, 114 (1), 5-14.

[3] FISHER, E. S.; WENNBERG, D. E.; STUKEL, T. A.; GOTTLIEB D. J.; LUCAS F. L. y PINDER, E. L. (2003a). «The implications of regional variations in Medicare spending. Part 1: the content, quality, and accessibility of care». Annals of Internal Medicine, 138 (4), 273-87.

[4] FISHER, E. S.; WENNBERG, D. E.; STUKEL, T. A.; GOTTLIEB, D. J.; LUCAS, F. L. y PINDER, E. L. (2003b). «The implications of regional variations in Medicare spending. Part 2: health outcomes and satisfaction with care». Annals of Internal Medicine, 138 (4), 288-98.

[5] FISHER, E. S. y WENNBERG, J. E. (2003). «Health care quality, geographic variations, and the challenge of supply-sensitive care». Perspectives in Biology and Medicine, 46 (1), 69-79.

[6] FROHLICH, N. y ROOS. N. P. (2002). «Searching for El Dorado: the impossibility of finding the right rate». Canadian Medical Association Journal, 167 (8), 880-1.

[7] GORGEMANS, S.; COMENDEIRO-MAALøE, M.; RIDAO-LÓPEZ, M. y BERNAL-DELGADO, E. (2018). «Quality and technical efficiency do not evolve hand in hand in Spanish hospitals: Observational study with administrative data». PLoS One, 13 (8), e0201466. 
[8] LIBRERO, J.; PEIRÓ, S.; BERNAL-DELGADO, E.; ALLEPUZ, A.; RIDAO, M.; MARTÍNEZ, N. y POR EL GRUPO VPM-IRYSS (2009). «Metodología del atlas de variaciones en hospitalizaciones por cirugía oncológica en el Sistema Nacional de Salud». Atlas de Variaciones en la Práctica Médica en el Sistema Nacional de Salud, 3 (2), 274-282.

[9] MINISTERIO DE SANIDAD, CONSUMO Y BIENESTAR SOCIAL (2018). «Estadística de Gasto Sanitario Público (EGSP)». Accesible en: http://www.mscbs.gob.es/ estadEstudios/estadisticas/inforRecopilaciones/gastoSanitario2005/home.htm.

[10] NHSENGLAND(2018).«Atlases».Accesibleen:https://www.england.nhs.uk/rightcare/ products/atlas/.

[11] PEIRÓ, S. y MAYNARD, A. (2015). «Variations in health care delivery within the European Union». European Journal of Public Health, 25, Suppl. 1, 1-2.

[12] PEIRÓ, S.; MENEU, R. y BERNAL, E. (2005). «Tres tristes tópicos sobre las variaciones en la práctica médica». Gestión Clínica y Sanitaria, 7, 47-51. Accesible en: http:// www.iiss.es/gcs/gestion24.pdf.

[13] RIDAO-LÓPEZ, M.; COMENDEIRO-MAALØE, M.; MARTÍNEZ-LIZAGA, N. y BERNAL-DELGADO, E. (2018). «Evolution of public hospitals expenditure by healthcare area in the Spanish National Health System: the determinants to pay attention to». BMC Health Services Research, 18 (1), 696.

[14] SIROVICH, B. E.; GOTTLIEB, D. J.; WELCH, H. G. y FISHER, E. S. (2006). «Regional variations in health care intensity and physician perceptions of quality of care». Annals of Internal Medicine, 144 (9), 641-9.

[15] VPM ATLAS DE VARIACIONES EN LA PRÁCTICA MÉDICA EN EL SISTEMA NACIONAL DE SALUD (2018). «Atlas de variaciones en la práctica médica». Accesible en: http://www.atlasvpm.org/atlas-variaciones-practica-medica.

[16] WENNBERG, J. E. y GITTELSOHN, A. (1973). «Small area variations in health care delivery». Science, 182 (4117), 1102-8.

[17] WENNBERG, J. E. y GITTELSOHN, A. (1982). «Variations in medical care among small áreas». Scientific American, 246 (4), 120-34.

[18] WENNBERG, J. E.; BARNES B. A. y ZUBKOFF, M. (1982). «Professional uncertainty and the problem of supplier-induced demand». Social Science and Medicine, 16 (7), 811-24.

[19] WENNBERG, J. E. (2010). Tracking medicine: a researcher's quest to understand health care». Nueva York, Oxford University Press.

[20] WENNBERG, J. E. (2011). «Time to tackle unwarranted variations in practice». British Medical Journal, 342, d1513. 


\section{ANEXO \\ CÓDIGOS DE SELECCIÓN DE DIAGNÓSTICOS Y PROCEDIMIENTOS}

\section{ATENCIÓN OBSTÉTRICA}

Sin restricciones de edad, pero excluye hombres. Incluye todos los procesos obstétricos (embarazo, parto y puerperio) y sus complicaciones.

Códigos CIE9MC: 630 a 679.

\section{APENDICECTOMÍA}

Sin restricciones de edad. Incluye todas las apendicectomías, incluso laparoscópicas e incidentales.

Códigos CIE9MC: 47.0, 47.1.

\section{FRACTURA DE CADERA}

Personas de 50 y más años.

Códigos CIE9MC: 820.0, 820.00, 820.01, 820.02, 820.03, 820.09, 820.1,820.10, $820.11,820.12,820.13,820.19,820.2,820.20,820.21,820.22,820.3,820.30$, $820.31,820.32,820.8,820.9$.

\section{COLECISTECTOMÍA}

Personas de 20 y más años. Incluye la colecistectomía abierta y laparoscópica.

Códigos CIE9MC: 51.22, 51.23.

\section{ARTROPLASTIA DE RODILLA}

Personas de 45 y más años. Incluye los procedimientos de reemplazo total o parcial de rodilla y la revisión de reemplazo de rodilla. Excluye los casos que se acompañan de un diagnóstico concomitante de fracturas y/o accidente.

Códigos CIE9MC: 81.54, 81.55, 00.80, 00.81, 00.82, 00.83, 00.84

\section{AMIGDALECTOMÍA CON O SIN ADENOIDECTOMÍA}

Personas menores de 20 años.

Códigos CIE9MC: 28.2, 28.3. 


\section{HOSPITALIZACIONES PREVENIBLES}

Personas de 40 y más años. Incluye:

- Complicaciones a corto de la diabetes. Códigos CIE9MC: 250.10250 .11 $250.2 * 250.3 *$

- EPOC. Códigos CIE9MC: 491.1, 491.2*, 491.8, 491.9, 492*, 493.2*, 494*, 496

- Insuficiencia Cardíaca Congestiva. Códigos CIE9MC: 398.91402 .01402 .11 402.91404 .01404 .03404 .11404 .13404 .91404 .93428 .0428 .1428 .20428 .22 428.23428 .30428 .32428 .33428 .40428 .42428 .43428 .9

- Deshidratación. Códigos CIE9MC: 276.0, 276.1, 276.5*, (276.50, 276.51, 276.52)

- Ángor sin procedimiento acompañante. Códigos CIE9MC: 411.1, 411.8*, $413 *(413.0,413.1,413.9)$;

- Asma. Códigos CIE9MC: 493.00, 493.01, 493.02, 493.10, 493.11, 493.12, 493.81, 493.82, 493.90, 493.91, 493.92518 .81 (solo si van acompañados de alguno de los siguientes diagnósticos secundarios: 493.00, 493.01, 493.02, 493.10, 493.11, 493.12, 493.81, 493.82, 493.90, 493.91, 493.92). 